\title{
Article \\ Alleviation of Osteoarthritis-Induced Pain and Motor Deficits in Rats by a Novel Device for the Intramuscular Insertion of Cog Polydioxanone Filament
}

\author{
Kilyong Lee ${ }^{1}$, Geung Gyu Gang ${ }^{2}$, Yun Gyu Kang ${ }^{2}$, Sung Sam Jung ${ }^{3}{ }^{\circledR}$, Hee-Gon Park ${ }^{4}$ and Jun Ho Jang ${ }^{2, *}$ \\ 1 Spine Center, Seoul Yes Hospital, Yongin 16868, Gyeonggi-do, Korea; yeskylee2@gmail.com \\ 2 Research Institute, OV MEDI Co., Ltd., Seongnam 13494, Gyeonggi-do, Korea; ceo@ovmedi.com (G.G.G.); \\ braintower@ovmedi.com (Y.G.K.) \\ 3 Neurosurgery Department, Yonseibarean Neurosurgery Clinic, Anyang 14001, Gyeonggi-do, Korea; \\ sungsamj@gmail.com \\ 4 Department of Orthopedic Surgery, Dankook University College of Medicine, \\ Cheonan 31116, Chungcheongnam-do, Korea; osdku@dankook.ac.kr \\ * Correspondence: junho@ovmedi.com
}

check for

updates

Citation: Lee, K.; Gang, G.G.; Kang, Y.G.; Jung, S.S.; Park, H.-G.; Jang, J.H. Alleviation of Osteoarthritis-Induced Pain and Motor Deficits in Rats by a Novel Device for the Intramuscular Insertion of Cog Polydioxanone Filament. Appl. Sci. 2021, 11, 10534. https://doi.org/10.3390/ app112210534

Academic Editors: Redha Taiar, Yuri Ivanenko and João R. Vaz

Received: 13 October 2021

Accepted: 7 November 2021

Published: 9 November 2021

Publisher's Note: MDPI stays neutral with regard to jurisdictional claims in published maps and institutional affiliations.

Copyright: (C) 2021 by the authors Licensee MDPI, Basel, Switzerland. This article is an open access article distributed under the terms and conditions of the Creative Commons Attribution (CC BY) license (https:// creativecommons.org/licenses/by/ $4.0 /)$.
Abstract: The importance of skeletal muscles in the development of osteoarthritis (OA) is known. However, in OA, strengthening the muscle is arduous process. This study investigated the effects of muscle enhancement and support therapy (MEST), a novel device for the intramuscular insertion of cog polydioxanone filament intended to hold and stimulate surrounding muscles, on OA-induced symptoms. In our results, the MEST attenuated OA-induced pain and mobility limitations, as evidenced by increases in withdrawal thresholds, rearing duration and travelled distance in an open cage, and fall latency from rotarod. It further restored atrophic rectus femoris muscle (RFM) in OA animals by increasing mass, decreasing nucleus density, and increasing the cross-sectional area of muscle fibers. Decreased collagen and insulin-like growth factor 1 levels in OA animals were restored without affecting the interleukin-6 and tumor necrosis factor-alpha levels in RFM. No evidence of structural improvement in the knee was observed via computed tomography after MEST. These results suggest that MEST in the quadriceps is effective for relieving pain and motor impairment in knee OA animals by restoring atrophic muscles, providing a novel therapeutic strategy for OA symptom management.

Keywords: osteoarthritis; skeletal muscle; pain; motor impairment; polydioxanone

\section{Introduction}

Osteoarthritis (OA) is the most common joint disease worldwide, with age-associated increases in both incidence and prevalence [1]. Debilitating chronic pain and reduced mobility, a leading symptom of OA, severely decreases the quality of life in approximately $10 \%$ of men and $18 \%$ of women aged $>60$ years worldwide [2]. Given its increasing prevalence and incidence, $\mathrm{OA}$ is now considered a major public health problem. The incidence of osteoarthritis increases with age, but it is not an inevitable consequence. Nevertheless, age is the strongest predictor [3]. Other notable risk factors include gender, race/ethnicity, bone density, postmenopausal estrogen decline, nutritional factors, and heredity. Biomechanical factors include obesity, quadriceps weakness, joint damage, joint trauma, and malformation [1]. Current treatments including pain relief, pharmaceutical drugs (e.g., non-steroidal anti-inflammatory drugs), lifestyle modifications (e.g., weight loss and exercise), intra-articular therapies, and surgical interventions are available to alleviate pain and improve joint function; however, their efficacy remains limited [2-4]. Thus, continuous efforts are needed to develop novel and effective therapeutic strategies.

A growing body of evidence indicates that skeletal muscles, which appear irrelevant to the function or pathology of the attached joint, play important roles in the induction and 
progression of OA [5,6]. A systematic review and meta-analysis showed that knee extensor muscle weakness is a risk factor for knee OA development [7]. Strategies to improve skeletal muscle health are increasingly recognized as important for improving symptomatic and functional outcomes in patients with knee OA. Lower limb muscle strengthening exercises are recommended by clinical practice guidelines as a core component of knee OA management $[6,8,9]$. Furthermore, a randomized clinical trial showed that quadricep strengthening exercises are effective in improving pain, function, and quality of life of patients with knee OA [10], and high-intensity resistance exercise demonstrated moderate quality of evidence for large and sustained improvements in knee muscle strength in patients with knee OA [11]. Although proper exercise is recommended by clinical guidelines as a key component for OA management [12-14], it is not always possible for patients with OA to perform such exercises to achieve muscle strengthening due to severe pain and weakened muscles.

In this study, we tested whether a new MEST device, which delivers specially designed polydioxanone (PDO) filaments to major skeletal muscles associated with OA, affects the symptoms of OA. PDO has gained increasing interest in the medical and pharmaceutical fields owing to its degradation to low-toxicity monomers in vivo. Previous animal studies have shown that PDO threads have the advantages of high safety, ease of procedure, high efficiency, and lower risk of side effects [15-17]. Therefore, the PDO led to stressshielding phenomena by unloading the autograft from tensional stimulation of fibroblasts in the tissue [18]. Paying attention to these points, in this study, we tried to confirm the improvement effect of OA symptoms using PDO filaments in OA-induced joint pain in terms of physical activity reduction and muscle weakness.

For this purpose, flexible filaments equipped with special cogs were used. Cog PDO filaments may effectively hold and support surrounding muscles to increase joint stability and relieve pain. Cog filaments may also stimulate muscles for physiological changes such as muscle growth when directly inserted into muscles. Inserted filaments are designed to be securely anchored to muscle tissue using cogs, whereas quadriceps keep the contract and relax repeatedly in daily movements. Sustained and repeated mechanical stimuli of surrounding muscles are anticipated by inserted and securely anchored $\operatorname{cog}$ PDO filaments. Although various absorbable threads have been used safely for suture and skin lifting [19], there is no information on intramuscular injection of PDO filaments and their effects on muscles and associated joint diseases. Thus, we investigated whether MEST of the quadriceps relieved OA-induced knee pain, improved mobility in vivo, and enhanced muscles. We further examined the effects of MEST on the local amount of collagen, insulinlike growth factor 1 (IGF-1), and pro-inflammatory cytokines (interleukin-6 [IL-6] and tumor necrosis factor- $\alpha$ [TNF- $\alpha]$ ) in vitro, as well as the structural changes confirmed via micro-computed tomography in OA knee joints. Our study shows that MEST-induced skeletal muscle changes may help alleviate the symptoms of OA.

\section{Materials and Methods}

\subsection{Animals}

Male Sprague Dawley rats (Taconic Biosciences; 6-7 weeks of age at the start of experiments) that were housed in groups of three to four, with food and water available ad libitum under a $12 \mathrm{~h}$ light/dark cycle, were used in this study. Animals were randomly assigned to the following treatment groups, and all experiments were performed under blinded conditions of the experimental groups. The sample sizes were determined based on previous studies that used rodent OA animals for similar experimental procedures [20,21]. (i) Naïve: no treatment $(\mathrm{n}=8)$; (ii) monosodium iodoacetate (MIA): rats were subjected to 1st and 2nd MIA injections on days 0 and $14(\mathrm{n}=7)$; (iii) MIA-MEST: same as the MIA group plus MEST on day $21(\mathrm{n}=8)$, (iv) MIA Needle: same as the MIA group plus sham needle treatment at day $21(\mathrm{n}=8)$. All experiments were conducted in accordance with the Guide for the Care and Use of Laboratory Animals, as adopted by the National Institutes of Health. The experimental protocols were approved by the Animal Care and Use Committee 
of the OV MEDI Co., Ltd. All methods were performed in accordance with the relevant guidelines and regulations and reported in accordance with the ARRIVE guidelines.

\subsection{Induction of $O A$ and MEST Application}

MIA-induced OA model is one of the most popular animal models, especially in pain mechanism research [22]. Rats were briefly anesthetized using isoflurane $/ \mathrm{O}_{2}$ inhalation and received an intra-articular injection of MIA (Sigma Aldrich, Milwaukee, WI, USA) in the left knee. MIA in a total volume of $30 \mu \mathrm{L}$ of physiological saline was injected through the intra-patellar ligament using a $26-\mathrm{G}$ needle. As motor behavioral deficits in OA animals were not evident at 2 weeks after the 1st MIA (1 mg) injection in our experimental protocols, an additional 2nd MIA injection $(3 \mathrm{mg}$ ) was administered similarly. MEST (MEST-B2375, OV MEDI, Korea) is a device for intramuscular insertion of specially designed polydioxanone filaments. It comprises a protective cap, needle (23-gauge, $75 \mathrm{~mm}$ ) with handle, a catheter (18-gauge, $35 \mathrm{~mm}$ ) with a handle, and flexible PDO filament (outer diameter $0.3 \mathrm{~mm}$ ) with cogs ( $1 \mathrm{~mm}$ intervals) (Figure 1A-D). The PDO filament, folded and inserted into two lines, is equipped with bi-directional cogs for maximal holding, supporting, and stimulating muscles (Figure 1C,D). Cogs in the opposite direction of filament insertion were covered by a catheter for protection (Figure 1D). Although the needle and catheter of MEST were designed to be fully inserted into the target muscles until the handle of the catheter reached the skin for clinical human use, some of them were inserted into muscles for this animal experiment (Figure 1F). Skin incision and closure were additionally performed for animal experiments because loose skin over the target muscles hindered the correct injection of the MEST needle to the intended points (Figure 1E-H).

The skin over the thigh muscles of the left hind paw was shaved and opened under brief anesthesia (isoflurane $/ \mathrm{O}_{2}$ inhalation) (Figure 1E). The MEST needle was inserted approximately $1 / 3$ of the entire length of the thigh muscles from the knee and advanced toward the knee at an angle of approximately $30^{\circ}$ below the horizontal (Figure 1F). Once the needle of MEST reached the distal femur area, the needle and catheter were withdrawn back sequentially, leaving the PDO filament fixed to the quadriceps muscles by the cogs. Five MESTs were administered at intervals across the lateral, medial, and ventral parts of the thigh muscles (Figure 1G). The skin was closed with 5-0 silk thread after removing the remaining part of the PDO filaments outside of the skin using scissors (Figure 1H). The skin was wiped with a povidone-iodine solution, and the animals were returned to their cages for recovery. Sham needling of MEST was performed similarly without the PDO filament.

\subsection{Behavioral Tests}

Mechanical withdrawal responses. Rats were acclimated to testing cages on a stainlesssteel mesh for $2 \mathrm{~h}$ per day for 3 consecutive days before starting the experiments, and $1 \mathrm{~h}$ immediately before each test. A series of von Frey filaments $(0.6,1.0,1.4,2.0,4.0,6.0,8.0$, and $10.0 \mathrm{~g}$ ) were applied for $1 \mathrm{~s}$ perpendicularly to the middle of the plantar surface of the left hind paw until each filament bent, starting with the $2.0 \mathrm{~g}$ stimulus. The $50 \%$ paw withdrawal threshold (PWT) was calculated using the simplified up-down method [23].

Spontaneous behaviors. The animals were placed in a transparent plastic cage $(28 \times 42 \times 18 \mathrm{~cm})$ without a lid and recorded from above and side simultaneously for $5 \mathrm{~min}$. The rearing duration was measured by counting the time spent on the two forepaws off the ground. The time spent grooming with the forepaws was excluded from the rearing duration. The distance covered by the animals from the center point was analyzed using video tracking software (EthoVision XT, Noldus, The Netherlands).

Rotarod test. Animals were placed on the rotarod apparatus (RRR, Scitech Korea, Seoul, South Korea) and trained once for $5 \mathrm{~min}$ at a constant speed of $4 \mathrm{rpm}$ before starting the experiments. The latencies to fall off the rotarod rotating under continuous acceleration (from 4 to $40 \mathrm{rpm}$ over $5 \mathrm{~min}$ ) were recorded with a $300 \mathrm{~s}$ cut-off time. 

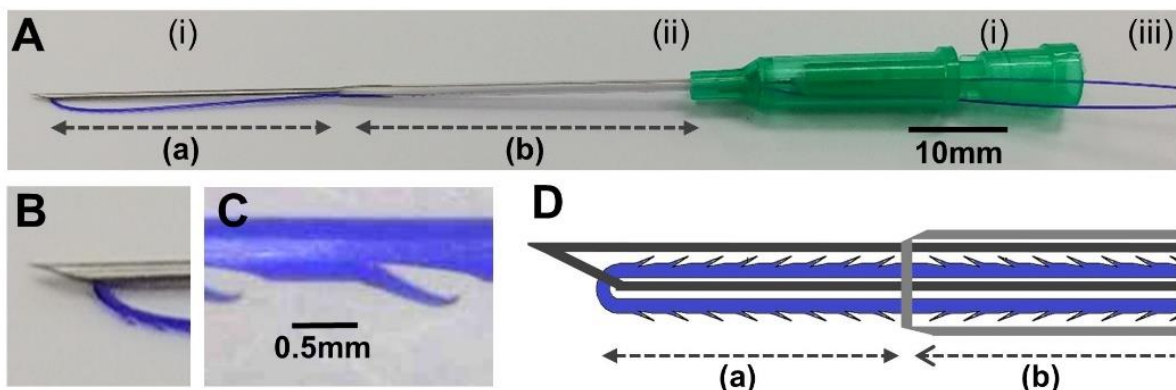

(b)
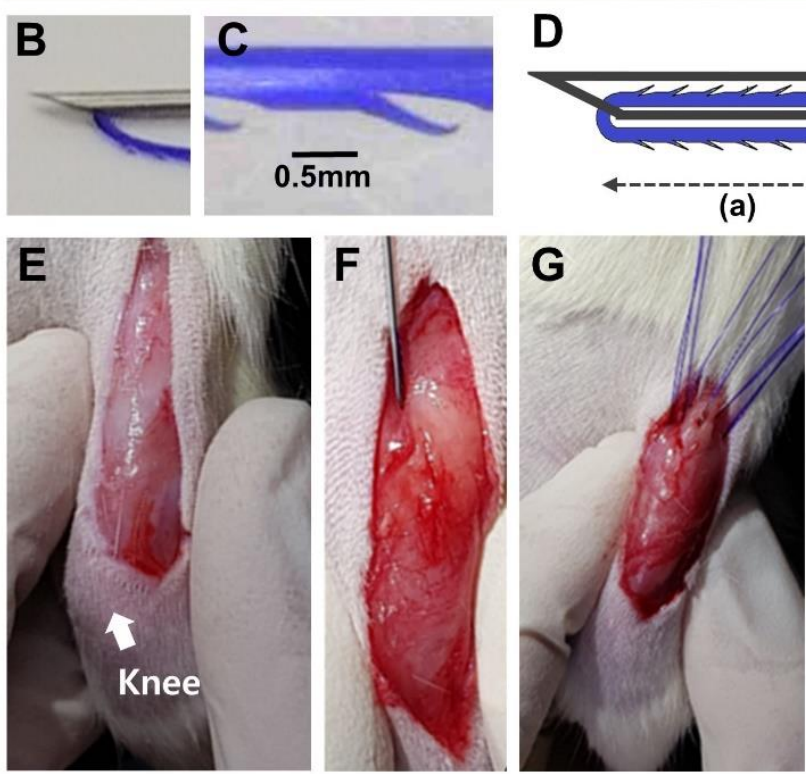

(b)

Figure 1. Application of MEST. MEST consists of (i) needle with handle, (ii) catheter with handle, (iii) PDO filament (A). The PDO filament equipped bi-directional cogs for effective muscle holding (B-D). The cogs in the forward direction (a) of needle insertion were exposed whereas cogs in the reverse direction (b) were protected by the catheter during needle insertion (D). After administration, the remaining filaments outside of the skin (G) were cut and removed. MEST was performed in rats similar to the purpose of clinical use, except that skin incision and closure were included under respiratory anesthesia. In addition, the needle was only partially inserted for rats due to the small size of the muscles (E-H).

After the behavioral experiments were completed, the RFMs of naïve, MIA, and MIA-MEST groups were extracted at day 56 or 87 for histological and molecular biological studies. The MIA-Needle group was not included in further studies because of the lack of effect observed in behavioral experiments. Additional animals were included in the naïve $(\mathrm{n}=5)$, MIA $(\mathrm{n}=4)$, and MIA-MEST $(\mathrm{n}=4)$ groups only for gross observation and micro-computed tomography (CT) of the knee joints performed on day 56 .

\subsection{Enzyme-Linked Immunosorbent Assay (ELISA)}

Commercially available ELISA kits were used to measure the levels of collagen (Sircol soluble collagen assay kit, Biocolor, Carrickfergus, UK) and IGF-1 (Quantikine ELISA Mouse/Rat IGF-1, R\&D Systems, Minneapolis, MN, USA) in the extracted rectus femoris muscle (RFM), one of the four quadriceps muscles of the left hind paw, according to the manufacturer's instructions.

\subsection{Western Blotting}

Tissue samples from the left RFM were homogenized in a lysis buffer. The protein concentration of the samples was determined using a bicinchoninic acid protein assay kit (Thermo Fisher Scientific, Waltham, MA, USA). Protein samples were homogenized and boiled $\left(5 \mathrm{~min}\right.$ at $95^{\circ} \mathrm{C}$ ) in Laemmli buffer, applied on $10 \%$ sodium dodecyl sulfate-polyacrylamide gel electrophoresis, and transferred to polyvinylidene fluoride membranes. These membranes were blocked with $5 \%(w / v)$ non-fat dry milk for $1 \mathrm{~h}$ at room temperature. The membranes were then incubated overnight at $4{ }^{\circ} \mathrm{C}$ with pri- 
mary antibodies (1:5000) for TNF- $\alpha$ (SCBT, Dallas, TX, USA) and IL-6 (R\&D Systems, Minneapolis, MN, USA). Bands were detected using an enhanced chemiluminescent solution (Thermo Fisher Scientific, Waltham, MA, USA) after incubation with an appropriate horseradish peroxidase-conjugated secondary antibody, and the bands were visualized using the Odyssey Fc imaging system (LiCor, Lincoln, Nebraska, USA). The intensities of the bands were quantified using Multi-Gauge software (Fuji Film, Tokyo, Japan).

\subsection{Histology}

Each extracted RFM was divided into several pieces and used for both transverse and longitudinal sections. Tissue samples were fixed in $4 \%$ paraformaldehyde (PFA) and $4 \%$ sucrose in phosphate-buffered solution (PBS) for $72 \mathrm{~h}$ at $4{ }^{\circ} \mathrm{C}$, kept in $30 \%$ sucrose in PBS for $72 \mathrm{~h}$ at $4{ }^{\circ} \mathrm{C}$, then embedded in optimal cutting temperature compound at $-80^{\circ} \mathrm{C}$ and cryostat-sectioned at $30 \mu \mathrm{m}$ thickness. The sections were then placed on glass slides and stained with hematoxylin and eosin using standard protocols [2]. The longitudinal sections were scanned at $40 \times$, using an Olympus BX51 microscope (Olympus, Tokyo, Japan), and the number of nuclei in the randomly selected area $\left(10^{6}\right.$ pixels $)$ of samples were counted. The transverse sections were scanned at $40 \times$ using a confocal microscope (Zeiss LSM 710, Carl Zeiss, Oberkochen, Germany), and the cross-sectional areas of five muscle fibers located in the center of the randomly selected area were measured. All image data were analyzed using MetaMorph software (Molecular Devices, San Jose, CA, USA).

\subsection{Gross Morphology}

As the rats were euthanized, the left knee joint was gently separated. After removing the excess soft tissue under a dissecting microscope and rinsing the articular surface of each bone with PBS, images of proximal tibias and distal femurs were captured.

\subsection{Micro-CT}

The whole left hind limb was dissected with the knee straight after cardiac perfusion with $4 \%$ PFA and stored in the same PFA solution at $4{ }^{\circ} \mathrm{C}$ until image acquisition. Micro-CT was performed on the knee joint using a Quantum FX scanner (Perkin-Elmer, USA) at an isotropic voxel size of $20 \mu \mathrm{m}$ (X-ray source voltage, $90 \mathrm{kVp}$; current, $160 \mu \mathrm{A} ; 10 \times 10 \mathrm{~mm}$ field of view; 3 min acquisition time). Morphometric analysis was performed using CTAn software v.1.13 (Bruker, USA) for the cancellous bone in the proximal tibial epiphysis (stack of 40 axial-sections; $0.8 \mathrm{~mm}$ height) to evaluate the following bone structural parameters: bone volume (BV), bone volume fraction (bone volume/total volume, BV/TV), trabecular number (Tb.N), trabecular separation (Tb.Sp), and bone mineral density (BMD).

\subsection{Statistical Analysis}

Behavioral data were analyzed using two-way mixed analysis of variance (ANOVA). Group differences at each time point were subsequently analyzed using Tukey's (three groups) or Sidak's (two groups) test. For other data, the differences among the three groups at specific time points were evaluated using either one-way ANOVA followed by Tukey's test or Kruskal-Wallis test followed by Dunn's test based on the Shapiro-Wilk test for normal distribution. Behavioral data are presented as mean \pm SEM and other data as means $\pm 95 \%$ confidence intervals (CI). Statistical significance was set at $p<0.05$. All data analyses were performed using Prism v.7.0 (GraphPad, USA) and SigmaPlot v.12.0 (Systat Software, USA) software.

\section{Results}

\subsection{Pain Relief and Motor Deficit Recovery Using MEST}

MIA-injected animals showed mechanical hypersensitivity, which started within a week after the 1st MIA injection, worsened later, and was maintained until day 56 compared to Naïve animals that showed stable PWTs throughout the entire experiment (Figure 2A). MEST on day 21 progressively reversed MIA-induced PWT reduction from day 35 to day 
56 compared to the MIA group. There was little change in rearing duration in Naïve animals until day 56 , whereas MIA-injected animals maintained a decreased duration on days 28,35 , and 56 (Figure 2B). MEST on day 21 recovered MIA-induced reduction in rearing duration from day 28 to day 56 compared to MIA-injected animals. The distance covered by Naïve animals tended to decrease until day 14 but stabilized thereafter until day 56 (Figure 2C). MIA-injected animals travelled less from day 28 to day 56 compared to Naïve animals. MEST administration on day 21 increased the distance of movement of MIA-injected animals on day 35 compared to the MIA group. MIA injection led to a decrease in the latency to fall off the rotarod from day 21 to day 56 compared to Naïve animals, which showed stable latency after the initial slight increase (Figure 2D). MEST on day 21 reversed MIA-induced reduction in fall latency on days 28, 42, and 56 compared to the MIA group. Sham needling of MEST performed on day 21 had no significant effects on PWT, rearing duration, covered distance, and fall latency of MIA-injected animals throughout the experiments (Supplementary Materials: Figure S1).

A

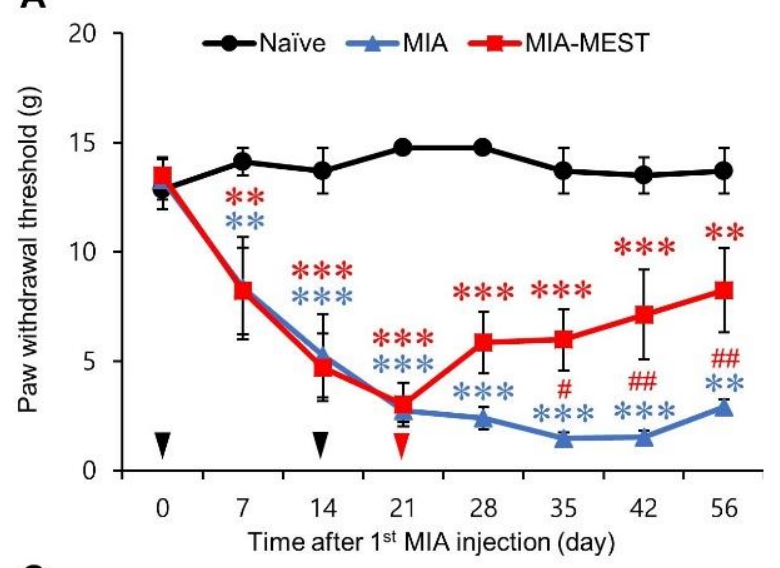

C

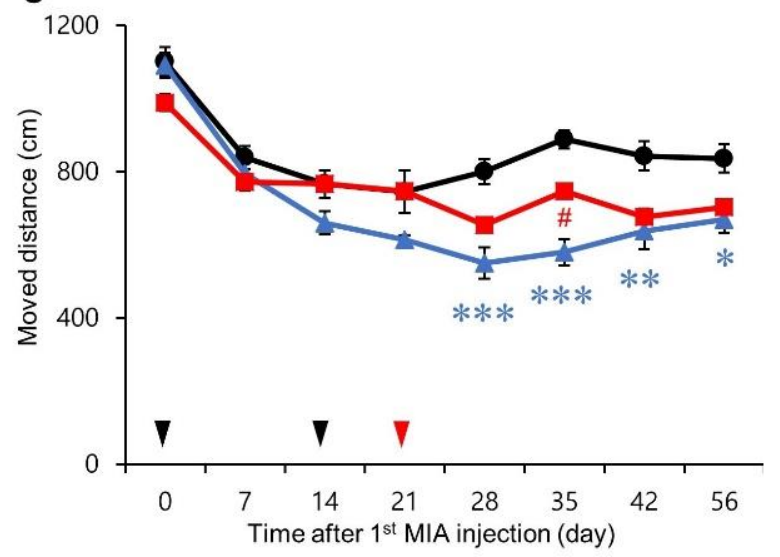

B

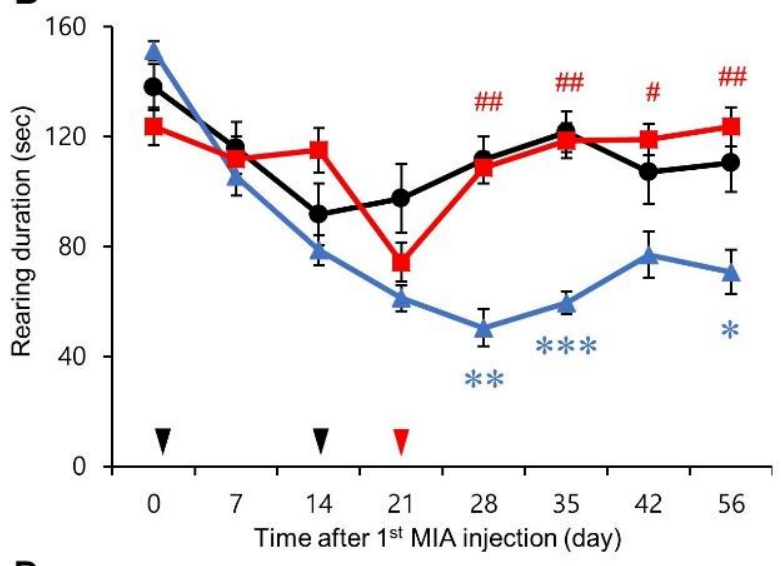

D

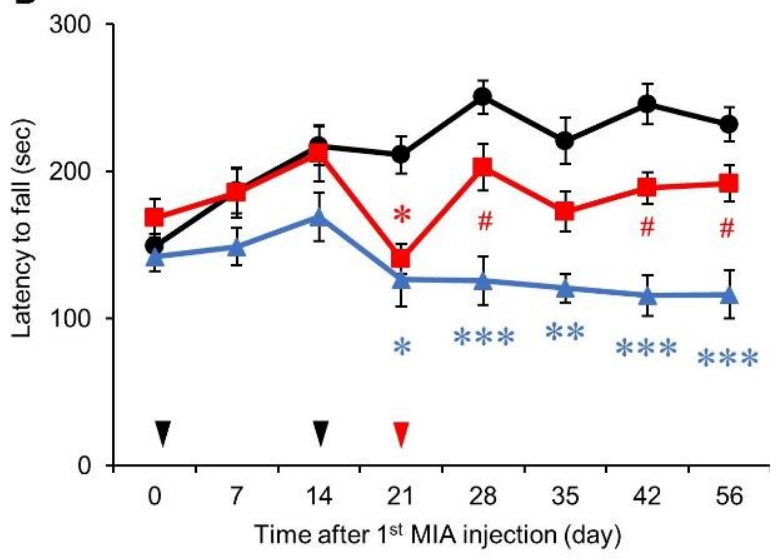

Figure 2. MEST alleviates MIA-induced pain and motor deficit. Paw withdrawal threshold to von Frey filaments (A). Time spent for rearing (B). Covered distance in an open cage (C). Fall latency from rotarod (D). Black and red arrowheads indicate MIA injections and MEST, respectively. ${ }^{*} p<0.05,{ }^{* *} p<0.01,{ }^{* * *} p<0.001$ vs. Naïve control. \# $p<0.05$, \#\# $p<0.01$ vs. MIA group. $\mathrm{N}=7-8$ per group. Data are presented as mean $\pm \mathrm{SEM}$.

\subsection{Restoration of Muscle Atrophy Using MEST}

MIA-injected animals showed decreased RFM weight/body weight (MW/BW) ratio on day 56 compared to Naïve animals $(p=0.0008)$ (Figure 3A), showing reduced muscle mass. MEST on day 21 recovered the MW/BW ratio of MIA-injected animals compared to MIA-injected animals on day 56 ( $p=0.0022)$ (Naïve, MIA, MIA-MEST; $0.366 \% \pm 0.016$, $0.258 \% \pm 0.074,0.348 \% \pm 0.017$, respectively). A similar pattern was observed on day 
87 (Naïve, MIA, MIA-MEST; $0.366 \% \pm 0.046,0.225 \% \pm 0.020,0.324 \% \pm 0.026$, respectively). The number of nuclei in the equal longitudinal area of RFM in the MIA group was higher than in the Naïve group on day $56(p<0.0001)$ (Figure 3B,C). MEST on day 21 reversed this longitudinal shrinkage of the RFM at day $56(p<0.0001)$ (Naïve, MIA, MIA-MEST; $51.310 \pm 3.867,82.814 \pm 5.444,63.047 \pm 3.348$, respectively). Similar findings were obtained on day 87 (Naïve, MIA, MIA-MEST; $54.684 \pm 4.927,85.474 \pm 7.519$, and $69.902 \pm 6.135$, respectively). Decreased transverse areas of muscle fibers of RFM were observed in the MIA-injected animals compared to Naïve animals on day $87(p=0.0011)$ (Figure 3D,E), indicating the cross-sectional shrinkage of RFM. MEST on day 21 recovered the thickness of RFM fibers of MIA-injected animals compared to the MIA-injected animals on day $87(p=0.0351)$ (Naïve, MIA, MIA-MEST; $10741.440 \pm 1281.424,7824.844 \pm 986.269$, $10548.290 \pm 1932.505$, respectively).

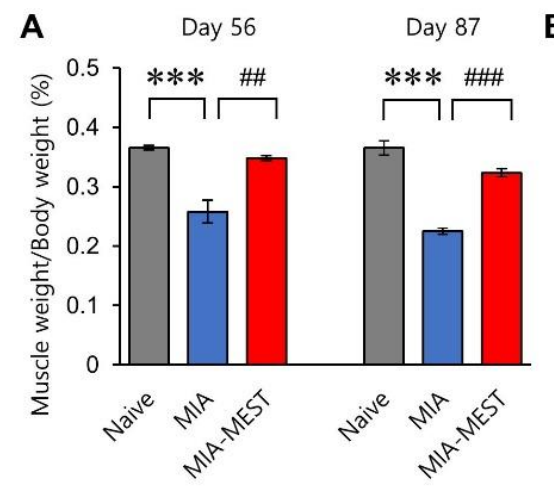

D

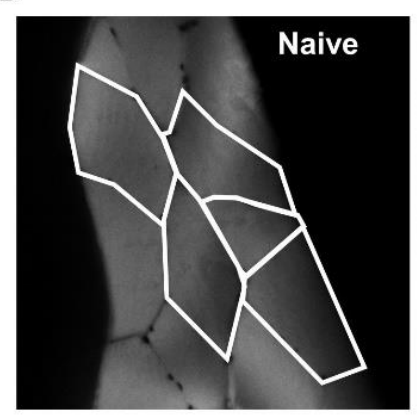

B
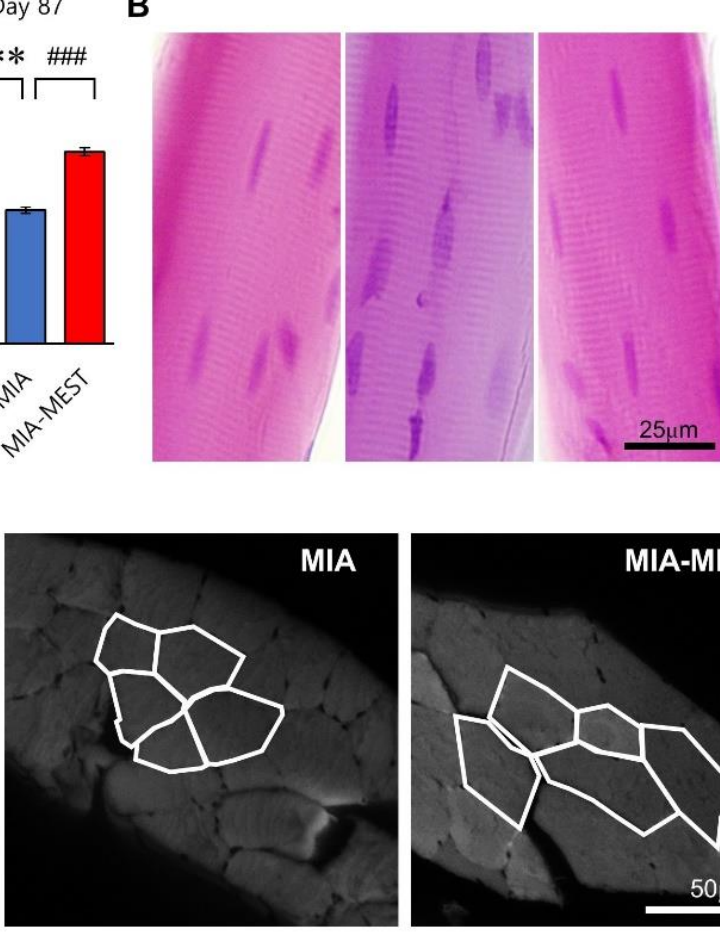

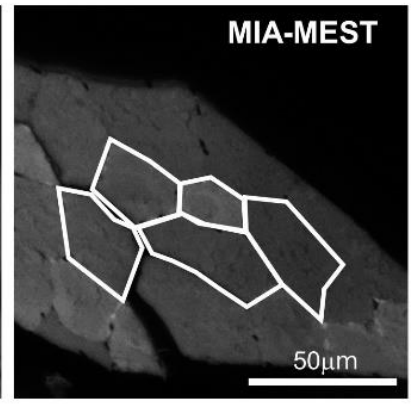

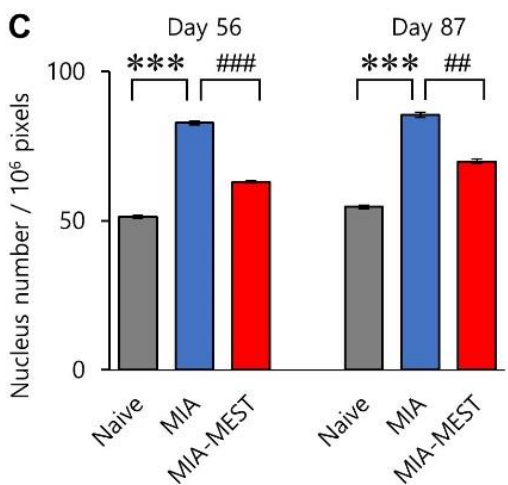

E

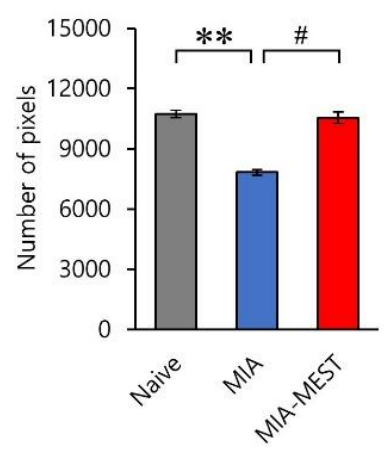

Figure 3. MEST alleviates MIA-induced muscle atrophy. The ratio of RFM weight to body weight on days 56 and 87 $(n=3)(A)$. Nucleus number in longitudinal section of RFM at days 56 and $87(n=3$, total 72 slices per group) (B,C). The cross-sectional area of muscle fibers in transverse section of RFM at day $87(\mathrm{n}=3$, total 45 cells per group) (D,E). Representative images on day 87 (B,D). White lines in D indicate the selected muscle fibers for data analysis. ${ }^{* *} p<0.01$, *** $p<0.001$. \# $p<0.05$, \#\# $p<0.01$, \#\#\# $p<0.001$. Data are presented as mean $\pm 95 \%$ confidence intervals.

\subsection{Recovery of Collagen and IGF-1 Levels Using MEST}

Compared to the Naïve group, collagen levels in RFM were decreased in the MIA group on day $56(p=0.0010)$ (Figure $4 \mathrm{~A})$. The MEST treatment performed at day 21 increased collagen levels of MIA-injected animals on day 56 compared to the MIA group ( $p=0.0086)$ (Naïve, MIA, MIA-MEST; $4.701 \pm 1.891,1.909 \pm 0.275,3.740 \pm 0.848$, respectively). The same pattern was observed on day 87 (Naïve, MIA, MIA-MEST; $4.554 \pm 0.201$, $1.878 \pm 0.555,4.449 \pm 0.638$, respectively). A decreased level of local IGF-1 was observed in the RFM of the MIA group vs. Naïve group ( $p=0.0059$ ) (Figure $4 B$ ). MEST on day 21 recovered IGF-1 levels in MIA-injected animals on day 87 vs. MIA animals $(p=0.0443)$ (Naïve, MIA, MIA-MEST; $108.896 \pm 43.086,61.3721 \pm 5.894,91.5177 \pm 25.000$ ). 

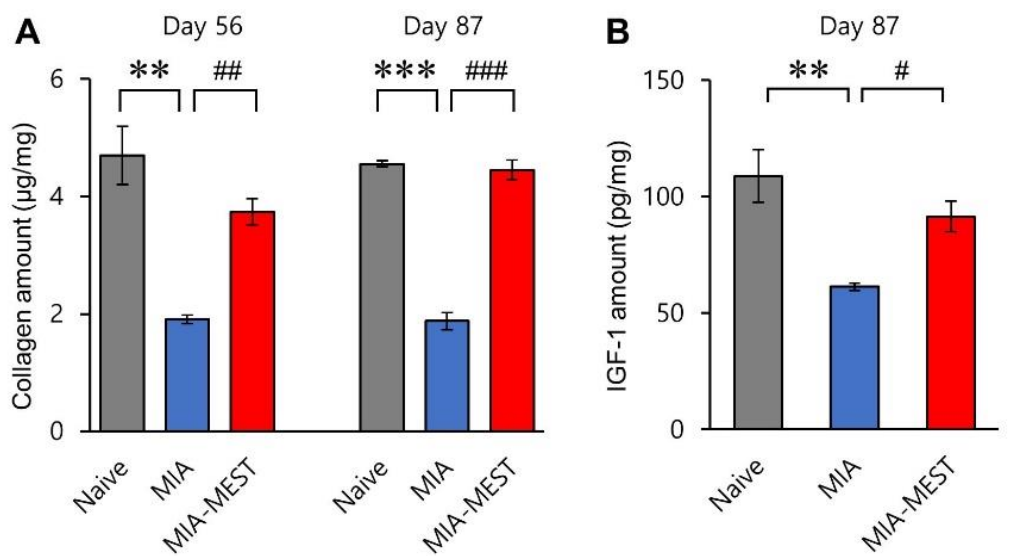

Figure 4. MEST restores MIA-induced decrease in collagen and IGF-1 levels. Local collagen level (A). Local IGF-1 levels in RFM on days 56 and 87 (B). ${ }^{* *} p<0.01,{ }^{* * *} p<0.001$. \# $p<0.05, \# \# p<0.01$, $\# \#$ \# $<0.001$. Data are presented as mean $\pm 95 \%$ confidence intervals.

\subsection{No Inflammatory Response Using MEST}

To evaluate the potential side effects of MEST, we measured the levels of IL-6 and TNF- $\alpha$ in RFM. Neither joint injection of MIA nor MEST in the quadriceps affected the normalized amount of IL-6 on day 56 (Naïve, MIA, MIA-MEST; $1.000 \pm 0.0000,1.013 \pm 0.0396$, $0.9534 \pm 0.0248$, respectively; $p=0.0704$ ) and 87 (Naïve, MIA, MIA-MEST; $1.000 \pm 0.0000$, $1.008 \pm 0.056,0.9528 \pm 0.050$, respectively; $p=0.3524$ ) (Figure 5B). TNF- $\alpha$ levels showed a similar pattern on day 56 (Naïve, MIA, MIA-MEST; $1.000 \pm 0.0000,0.9325 \pm 0.0272$, $0.9916 \pm 0.0957$, respectively; $p=0.4332$ ) and 87 (Naïve, MIA, MIA-MEST; $1.000 \pm 0.0000$, $1.01 \pm 0.0695,1.011 \pm 0.0904$, respectively; $p=0.9746$ ) (Figure $5 \mathrm{C}$ ).
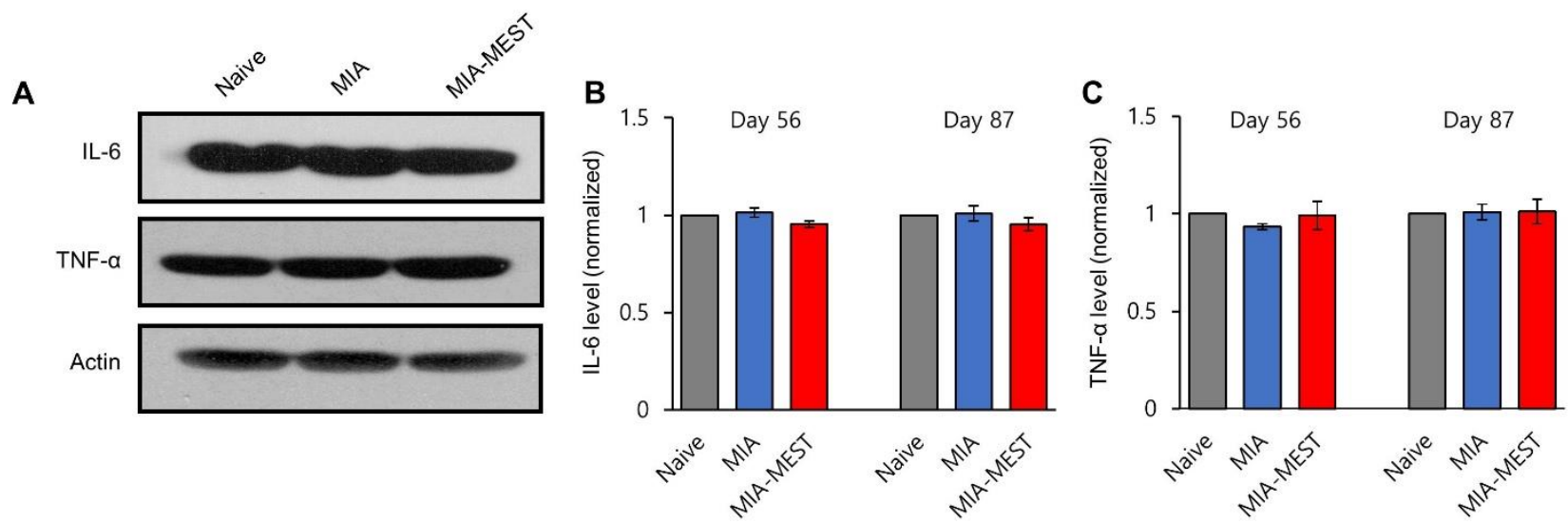

Figure 5. MEST does not affect the local amount of pro-inflammatory cytokines. Representative band images on day 87 (A). IL-6 and TNF- $\alpha$ levels in RFM on days 56 and $87(\mathbf{B}, \mathbf{C}) . \mathrm{N}=3$ per group. Data are presented as mean $\pm 95 \%$ confidence intervals.

\subsection{Effect of MEST on the Joint Structure}

Gross observation of the knee joint on day 56 (Figure 6A) revealed a smooth and shiny articular surface of the femoral condyles and tibial plateau in the Naïve group. In the MIA-injected knee, the cartilage surface almost disappeared, and the damaged subchondral bone was exposed. The MIA-MEST group showed fewer lesions in some cases on the articular surface than the MIA group (Figure 6A); however, the observations were not consistent across individual animals. Representative micro-CT images (Figure 6B) show joint deformation and destruction in the MIA group on day 56 compared to Naïve group. Similar images were acquired for the MIA-MEST group. Micro-CT analysis of the proximal 
tibial epiphysis on day 56 (Figure 6C-G) revealed decreased BV, BV/TV, Tb.N, and BMD and increased Tb.Sp in the MIA group compared to the Naïve group (Naïve, MIA; BV, $9.856 \pm 1.334,3.189 \pm 0.826, p<0.0001 ; \mathrm{BV} / \mathrm{TV}, 51.533 \pm 8.019,27.631 \pm 5.794, p<0.0001$; Tb.N, $2.741 \pm 0.308,1.590 \pm 0.334, p<0.0001 ;$ Tb.Sp $, 0.285 \pm 0.050,0.416 \pm 0.089, p=0.0067 ;$ $\mathrm{BMD}, 621.547 \pm 99.075,332.997 \pm 71.003, p<0.0001)$. Compared to animals in the MIA group, MEST on day 21 had no effects on BV, BV/TV, Tb.N, Tb.Sp, and BMD in MIAinjected animals on day 56 (BV, $p=0.4868 ; \mathrm{BV} / \mathrm{TV}, p=0.8019 ; \mathrm{Tb} . \mathrm{N}, p=0.6860 ; \mathrm{Tb} . \mathrm{Sp}$, $p=0.6515$, BMD, $p=0.7657$ ).
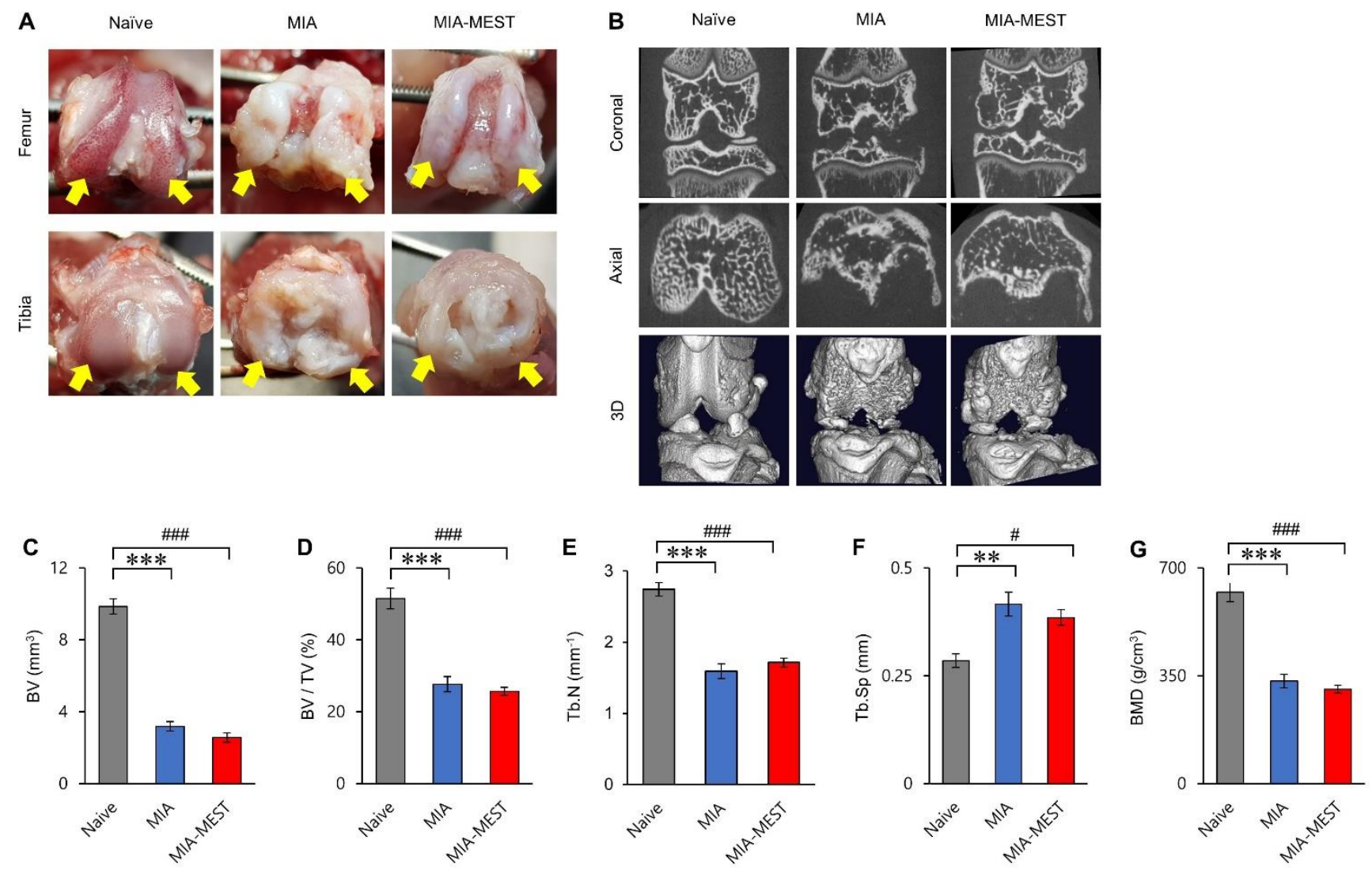

Figure 6. MEST does not affect the joint structure. Representative images of the knee joint on day 56 (A). The severe joint lesion was obvious in the MIA-injected knee; however, the structural improvement using MEST was inconsistent across individual animals via gross observation. Yellow arrows indicate femoral condyles and tibial plateau. Representative micro-CT images of the knee joint (B) and micro-CT analysis of the proximal tibial epiphysis (C-G) on day 56 . BV, bone volume; $\mathrm{BV} / \mathrm{TV}$, bone volume/total volume, Tb.N, trabecular number; Tb.Sp, trabecular separation; BMD, bone mineral density. ${ }^{* *} p<0.01,{ }^{* * *} p<0.001$. \# $p<0.05$, \#\#\# $p<0.001$. Data are presented as mean $\pm 95 \%$ confidence intervals.

\section{Discussion}

In this study, we demonstrated that intramuscular insertion of PDO filaments into the quadriceps using MEST relieves OA-induced pain and mobility limitations in vivo. Through the experiment, we also confirmed that MEST restored the reductions in RFM mass and local amounts of collagen and IGF-1 levels in OA animals without an inflammatory response. However, this recovery was not accompanied by structural changes in the knee OA. These results strongly suggest that the MEST application method for OA is very effective in reducing pain and improving athletic performance.

For many years, various types of threads made of PDO have been used for surgery and, more recently, for skin rejuvenation [19]. In particular, cog threads are widely used for cosmetic purposes, because they have barbs that hook and hold the tissue underneath the skin to lift sagging tissue [24,25]. In this study, we expanded the scope of their current 
applications to muscles and related joint diseases using MEST, which allow safe and simple intramuscular delivery of cog PDO filaments. To hold skeletal muscles effectively, various factors related to the filament (e.g., thickness, length, and degree of flexibility), equipped cogs (e.g., length, angle, spatial distribution, and direction), and delivery methods (e.g., cog protection during insertion, insertion angle, and insertion points) were carefully considered based on preliminary trials (data not shown). For instance, if the cogs are too long or too short, they become too flexible to hook and support muscles and will not be able to hook muscles efficiently. Moreover, it is crucial to keep the cogs in the opposite direction of insertion to avoid ruining during needle insertion using a protective catheter (Figure 1), because only well-preserved bi-directional cogs prevent filaments from moving around by providing firm anchoring to the tissue to avoid thread exposure or other unexpected serious side effects. MEST technique may work against the forces of vectors that generate tension and reconstruct tissue and provide efficient tension to the muscles, as suggested in skin rejuvenation technique using absorbable sutures [26,27].

Our behavioral tests results showed that a single treatment of MEST reversed MIAinduced mechanical pain in a week and maintained it throughout the entire experiment. In addition, decreased locomotor and exploratory activities and fall latency from rotarod, which reflect the existence of pain [28] and/or motor impairment [29], were successfully reversed. The changes in weight, nucleus density, and fiber thickness of RFM by MEST and no inflammatory response indicate the recovery of knee OA-induced quadriceps atrophy. MEST provides evidence of pain relief and improved mobility in OA. Although MEST administration in quadriceps did not bring a fundamental change in OA joint, the absence of structural improvement of OA joint was not surprising, as pain and loss of function are weakly correlated to the severity of OA by radiography in some cases [30].

Quadriceps weakness, which is commonly observed in patients with knee OA and considered a risk factor for the development of knee OA [5-8], may reduce the stability of the knee joint and the ability to absorb the shock transmitted to the joint $[7,8,31,32]$. This muscle weakness worsens the pain and immobility of patients with OA who already have impaired shock-absorbing properties of the knee joint due to the injured cartilage [33] and malalignment and abnormal loading at the patellofemoral joint [34] to form a vicious cycle, that is, joint pain-decreased movement-muscle weakness-decreased stability of joint-more pain. Although it is clear that changes in skeletal muscles are important players in OA and its symptoms [5-8,10,35-37], there are few methods to strengthen muscles such as exercise or physical therapy. Additionally, these methods are not always possible for patients to perform due to atrophic muscles and severe joint pain, especially in older people. Furthermore, the degree of benefits of exercise for patients with OA varies widely [38-41], and inappropriate exercises that may involve excessive mechanical stress on the already injured OA joint are even harmful [35]. Thus, MEST may provide an alternative or complementary approach to overcome the current limitations of exercise and physical therapy for OA management by directly regulating specific skeletal muscles related to the OA joint. When the muscles are stronger, they absorb some of the force that would otherwise go through the joint, help relieve pain, and keep the mechanics of the joint working properly.

Given that the architecture and metabolism of skeletal muscles are highly sensitive to the mechanical environment [42], the inserted cog filaments may trigger skeletal muscle plasticity by directly stimulating muscle cells. Mechanical stimuli (e.g., an increase in cell membrane tension through stretching, deformation, or curvature) may not only lead to ion influx through multiple mechanoreceptors expressed on muscle cells to initiate various intracellular signaling pathways [42-45] but also local production of signaling molecules and growth factors $[42,46,47]$ that affect muscle growth, adaptation, and maintenance. For example, it is well known that mechanical stress on muscle cells induces the local release of IGF-1 [42,48,49], a key regulator of overall skeletal muscle mass, to initiate the $\mathrm{PI} 3 \mathrm{~K} / \mathrm{AKT} / \mathrm{mTOR}$ pathway to promote muscle growth in an autocrine/paracrine manner [49-52]. IGF-1 can stimulate the local synthesis of collagen [53] which leads to my- 
ofiber hypertrophy $[54,55]$ for muscle building. It has also been suggested that consuming collagen supplements in combination with exercise further boosted muscle growth and strength in people with age-related muscle mass loss [56]. Thus, the mechano-transduction mechanism [46], by which cells convert mechanical information into biochemical signals that elicit adaptive responses, may explain muscle enhancement (Figure 3) and IGF-1 and collagen levels (Figure 4) using MEST in OA animals.

Additional mechanisms might be necessary to explain the rapid onset of pain relief, and motor improvement started a week after MEST (Figure 2). For instance, similar to the suggested working mechanisms of kinesiology taping $[57,58]$ or unloader brace $[59,60]$ for OA management, the filaments inserted across the quadriceps could secure surrounding muscles with cogs to support muscle, improve the imbalance of muscle strength, and increase the stability of the knee joint, thereby resulting in less pain and better movement.

Taken together, minimally invasive MEST in the quadriceps is safe, rapid, and effective for relieving pain and motor impairment in MIA-induced knee OA animals. This may provide a novel therapeutic strategy for OA symptom management, but not for the direct treatment of OA. Further studies are needed to complement our findings. For examples, combining MEST with other conventional treatments, such as medication, joint injection, or surgery, may be even more effective than existing methods in terms of OA symptom management. It would be also interesting to compare the effects of MEST in different OA animal models [61], as various reasons cause OA in clinical settings. Apoptosis [62] and proliferation [63] staining data together will support the rationale for the suggested IGF-1 and PI3K/Akt pathway for muscle recovery by MEST.

Supplementary Materials: The following are available online at https:/ / www.mdpi.com/article / 10.3390/app112210534/s1. Figure S1: Sham needling of MEST does not affect MIA-induced pain and motor deficits.

Author Contributions: Conceptualization, G.G.G., Y.G.K., K.L. and J.H.J.; formal analysis and investigation, K.L., S.S.J., H.-G.P. and J.H.J.; resources, G.G.G. and Y.G.K.; supervision and writing, J.H.J. All authors have read and agreed to the published version of the manuscript.

Funding: This research received no external funding.

Institutional Review Board Statement: This study was approved by The Animal Care and Use Committee at the OV MEDI Co., Ltd. (OV2020F-02).

Informed Consent Statement: Not applicable.

Data Availability Statement: All data generated or analyzed during this study are included in this published article and its Supplementary Information Files.

Conflicts of Interest: G.G.G., Y.G.K. and J.H.J. are full-time employees of OV MEDI Co., Ltd. at the time of submission of this manuscript. All the other authors declare that they have no competing interests.

\section{References}

1. Healey, E.L.; Main, C.J.; Ryan, S.; McHugh, G.A.; Porcheret, M.; Finney, A.G.; Morden, A.; Dziedzic, K.S. A nurse-led clinic for patients consulting with osteoarthritis in general practice: Development and impact of training in a cluster randomised controlled trial. BMC Fam. Pract. 2016, 17, 173. [CrossRef]

2. Nelson, A. Osteoarthritis year in review 2017: Clinical. Osteoarthr. Cartil. 2018, 26, 319-325. [CrossRef]

3. Glyn-Jones, S.; Palmer, A.J.R.; Agricola, R.; Price, A.J.; Vincent, T.; Weinans, H.; Carr, A.J. Osteoarthritis. Lancet 2015, $386,376-387$. [CrossRef]

4. Martel-Pelletier, J.; Barr, A.; Cicuttini, F.; Conaghan, P.; Cooper, C.; Goldring, M.B.; Goldring, S.R.; Jones, G.; Teichtahl, A.J.; Pelletier, J.-P. Osteoarthritis. Nat. Rev. Dis. Prim. 2016, 2, 16072. [CrossRef]

5. Shorter, E.; Sannicandro, A.J.; Poulet, B.; Goljanek-Whysall, K. Skeletal Muscle Wasting and Its Relationship with Osteoarthritis: A Mini-Review of Mechanisms and Current Interventions. Curr. Rheumatol. Rep. 2019, 21, 1-8. [CrossRef]

6. Krishnasamy, P.; Hall, M.; Robbins, S. The role of skeletal muscle in the pathophysiology and management of knee osteoarthritis. Rheumatology 2018, 57, iv124. [CrossRef] [PubMed]

7. Øiestad, B.; Juhl, C.; Eitzen, I.; Thorlund, J. Knee extensor muscle weakness is a risk factor for development of knee osteoarthritis. A systematic review and meta-analysis. Osteoarthr. Cartil. 2015, 23, 171-177. [CrossRef] [PubMed] 
8. Segal, N.A.; Glass, N. Is Quadriceps Muscle Weakness a Risk Factor for Incident or Progressive Knee Osteoarthritis? Physician Sportsmed. 2011, 39, 44-50. [CrossRef] [PubMed]

9. $\quad$ Fernandes, L.; Hagen, K.B.; Bijlsma, J.W.J.; Andreassen, O.; Christensen, P.; Conaghan, P.; Doherty, M.; Geenen, R.; Hammond, A.; Kjeken, I.; et al. EULAR recommendations for the non-pharmacological core management of hip and knee osteoarthritis. Ann. Rheum. Dis. 2013, 72, 1125-1135. [CrossRef]

10. Imoto, A.M.; Peccin, M.S.; Trevisani, V. Exercícios de fortalecimento de quadríceps são efetivos na melhora da dor, função e qualidade de vida de pacientes com osteoartrite do joelho. Acta Ortopédica Bras. 2012, 20, 174-179. [CrossRef]

11. Zacharias, A.; Green, R.; Semciw, A.; Kingsley, M.; Pizzari, T. Efficacy of rehabilitation programs for improving muscle strength in people with hip or knee osteoarthritis: A systematic review with meta-analysis. Osteoarthr. Cartil. 2014, 22, 1752-1773. [CrossRef]

12. McAlindon, T.E.; Bannuru, R.R.; Sullivan, M.C.; Arden, N.K.; Berenbaum, F.; Bierma-Zeinstra, S.M.; Hawker, G.A.; Henrotin, Y.; Hunter, D.J.; Kawaguchi, H.; et al. OARSI guidelines for the non-surgical management of knee osteoarthritis. Osteoarthr. Cartil. 2014, 22, 363-388. [CrossRef]

13. Hochberg, M.C.; Altman, R.D.; April, K.T.; Benkhalti, M.; Guyatt, G.; McGowan, J.; Towheed, T.; Welch, V.; Wells, G.; Tugwell, P. American College of Rheumatology 2012 recommendations for the use of nonpharmacologic and pharmacologic therapies in osteoarthritis of the hand, hip, and knee. Arthritis Rheum. 2012, 64, 465-474. [CrossRef] [PubMed]

14. Zhang, W.; Moskowitz, R.W.; Nuki, G.; Abramson, S.; Altman, R.D.; Arden, N.; Bierma-Zeinstra, S.; Brandt, K.D.; Croft, P.; Doherty, M.; et al. OARSI recommendations for the management of hip and knee osteoarthritis, Part II: OARSI evidence-based, expert consensus guidelines. Osteoarthr. Cartil. 2008, 16, 137-162. [CrossRef]

15. Shin, J.J.; Park, T.J.; Kim, B.Y.; Kim, C.M.; Suh, D.H.; Lee, S.J.; Moon, H.-R.; Ryu, H.J. Comparative effects of various absorbable threads in a rat model. J. Cosmet. Laser Ther. 2018, 21, 158-162. [CrossRef] [PubMed]

16. Abhari, R.E.; Mouthuy, P.-A.; Zargar, N.; Brown, C.; Carr, A. Effect of annealing on the mechanical properties and the degradation of electrospun polydioxanone filaments. J. Mech. Behav. Biomed. Mater. 2017, 67, 127-134. [CrossRef] [PubMed]

17. Rashid, M.; Dudhia, J.; Dakin, S.G.; Snelling, S.; Lach, A.; De Godoy, R.; Mouthuy, P.-A.; Smith, R.; Morrey, M.; Carr, A.J. Histological evaluation of cellular response to a multifilament electrospun suture for tendon repair. PLoS ONE 2020, 15, e0234982. [CrossRef]

18. Peterson, L.; Ernest, C. 39-Augmented Grafts: Synthetic/Allograft/Autograft. In The Anterior Cruciate Ligament, 2nd ed.; Prodromos, C.C., Ed.; Elsevier: Amsterdam, The Netherlands, 2018; pp. 160-162.e161.

19. Tong, L.X.; Rieder, E. Thread-Lifts: A Double-Edged Suture? A Comprehensive Review of the Literature. Dermatol. Surg. 2019, 45, 931-940. [CrossRef] [PubMed]

20. Stevenson, G.W.; Mercer, H.; Cormier, J.; Dunbar, C.; Benoit, L.; Adams, C.; Jezierski, J.; Luginbuhl, A.; Bilsky, E. Monosodium iodoacetate-induced osteoarthritis produces pain-depressed wheel running in rats: Implications for preclinical behavioral assessment of chronic pain. Pharmacol. Biochem. Behav. 2011, 98, 35-42. [CrossRef] [PubMed]

21. Teixeira, V.D.O.N.; Filippin, L.I.; Viacava, P.R.; de Oliveira, P.G.; Xavier, R.M. Muscle wasting in collagen-induced arthritis and disuse atrophy. Exp. Biol. Med. 2013, 238, 1421-1430. [CrossRef]

22. Lampropoulou-Adamidou, K.; Lelovas, P.; Karadimas, E.V.; Liakou, C.; Triantafillopoulos, I.K.; Dontas, I.; Papaioannou, N.A. Useful animal models for the research of osteoarthritis. Eur. J. Orthop. Surg. Traumatol. 2014, 24, 263-271. [CrossRef]

23. Bonin, R.P.; Bories, C.; De Koninck, Y. A Simplified Up-Down Method (SUDO) for Measuring Mechanical Nociception in Rodents Using von Frey Filaments. Mol. Pain 2014, 10, 26. [CrossRef] [PubMed]

24. Kalra, R. Use of barbed threads in facial rejuvenation. Indian J. Plast. Surg. 2008, 41, 93-100. [CrossRef]

25. Myung, Y.; Jung, C. Mini-midface Lift Using Polydioxanone Cog Threads. Plast. Reconstr. Surg. Glob. Open 2020, 8, e2920. [CrossRef] [PubMed]

26. Tavares, J.D.P.; Oliveira, C.A.C.P.; Torres, R.P.; Bahmad, F. Facial thread lifting with suture suspension. Braz. J. Otorhinolaryngol. 2017, 83, 712-719. [CrossRef]

27. Sardesai, M.G.; Zakhary, K.; Ellis, D.A.F. Thread-lifts: The Good, the Bad, and the Ugly. Arch. Facial Plast. Surg. 2008, 10, $284-285$. [CrossRef]

28. Piel, M.J.; Kroin, J.S.; van Wijnen, A.J.; Kc, R.; Im, H.-J. Pain assessment in animal models of osteoarthritis. Gene 2014, 537, 184-188. [CrossRef]

29. Brooks, S.P.; Dunnett, S. Tests to assess motor phenotype in mice: A user's guide. Nat. Rev. Neurosci. 2009, 10, 519-529. [CrossRef]

30. McAlindon, T.E.; Cooper, C.; Kirwan, J.R.; Dieppe, P.A. Determinants of disability in osteoarthritis of the knee. Ann. Rheum. Dis. 1993, 52, 258-262. [CrossRef] [PubMed]

31. Hurley, M.V. The Role of Muscle Weakness in the Pathogenesis of Osteoarthritis. Rheum. Dis. Clin. North Am. 1999, 25, 283-298. [CrossRef]

32. Andriacchi, T.P.; Mündermann, A.; Smith, R.L.; Alexander, E.J.; Dyrby, C.O.; Koo, S. A Framework for the in Vivo Pathomechanics of Osteoarthritis at the Knee. Ann. Biomed. Eng. 2004, 32, 447-457. [CrossRef]

33. Shaktivesh; Malekipour, F.; Lee, P.V. Shock absorbing ability in healthy and damaged cartilage-bone under high-rate compression. J. Mech. Behav. Biomed. Mater. 2018, 90, 388-394. [CrossRef] [PubMed]

34. Macri, E.; Stefanik, J.J.; Khan, K.K.; Crossley, K.M. Is Tibiofemoral or Patellofemoral Alignment or Trochlear Morphology Associated With Patellofemoral Osteoarthritis? A Systematic Review. Arthritis Rheum. 2016, 68, 1453-1470. [CrossRef] [PubMed]

35. Hunter, D.J.; Eckstein, F. Exercise and osteoarthritis. J. Anat. 2009, 214, 197-207. [CrossRef] [PubMed] 
36. Juhl, C.; Christensen, R.; Roos, E.; Zhang, W.; Lund, H. Impact of Exercise Type and Dose on Pain and Disability in Knee Osteoarthritis: A Systematic Review and Meta-Regression Analysis of Randomized Controlled Trials. Arthritis Rheumatol. 2014, 66, 622-636. [CrossRef]

37. Luc-Harkey, B.A.; Safran-Norton, C.E.; Mandl, L.A.; Katz, J.N.; Losina, E. Associations among knee muscle strength, structural damage, and pain and mobility in individuals with osteoarthritis and symptomatic meniscal tear. BMC Musculoskelet. Disord. 2018, 19, 258. [CrossRef]

38. Hurley, M.; Dickson, K.; Hallett, R.; Grant, R.; Hauari, H.; Walsh, N.; Stansfield, C.; Oliver, S. Exercise interventions and patient beliefs for people with hip, knee or hip and knee osteoarthritis: A mixed methods review. Cochrane Database Syst. Rev. 2018, 4, CD010842. [CrossRef] [PubMed]

39. Schiphof, D.; Driest, J.J.V.D.; Runhaar, J. Osteoarthritis year in review 2017: Rehabilitation and outcomes. Osteoarthr. Cartil. 2018, 26, 326-340. [CrossRef]

40. Fransen, M.; McConnell, S.; Harmer, A.R.; Van Der Esch, M.; Simic, M.; Bennell, K. Exercise for osteoarthritis of the knee: A Cochrane systematic review. Br. J. Sports Med. 2015, 49, 1554-1557. [CrossRef]

41. Goh, S.-L.; Persson, M.S.M.; Stocks, J.; Hou, Y.; Welton, N.; Lin, J.; Hall, M.C.; Doherty, M.; Zhang, W. Relative Efficacy of Different Exercises for Pain, Function, Performance and Quality of Life in Knee and Hip Osteoarthritis: Systematic Review and Network Meta-Analysis. Sports Med. 2019, 49, 743-761. [CrossRef]

42. Tidball, J.G. Mechanical signal transduction in skeletal muscle growth and adaptation. J. Appl. Physiol. 2005, 98, 1900-1908. [CrossRef] [PubMed]

43. Martinac, B.; Cox, C.D. Mechanosensory Transduction: Focus on Ion Channels $九$. In Reference Module in Life Sciences; Elsevier: Amsterdam, The Netherlands, 2017; ISBN 978-0-12-809633-8. [CrossRef]

44. Vandebrouck, C.; Martin, D.; Schoor, M.C.-V.; Debaix, H.; Gailly, P. Involvement of TRPC in the abnormal calcium influx observed in dystrophic (mdx) mouse skeletal muscle fibers. J. Cell Biol. 2002, 158, 1089-1096. [CrossRef] [PubMed]

45. Krüger, J.; Kunert-Keil, C.; Bisping, F.; Brinkmeier, H. Transient receptor potential cation channels in normal and dystrophic mdx muscle. Neuromuscul. Disord. 2008, 18, 501-513. [CrossRef] [PubMed]

46. Thomas, J.B. Mechanotransduction in skeletal muscle. Front. Biosci. 2007, 12, 174-191. [CrossRef]

47. Humphrey, J.D.; Dufresne, E.R.; Schwartz, M.A. Mechanotransduction and extracellular matrix homeostasis. Nat. Rev. Mol. Cell Biol. 2014, 15, 802-812. [CrossRef]

48. Perrone, C.E.; Fenwick-Smith, D.; Vandenburgh, H.H. Collagen and Stretch Modulate Autocrine Secretion of Insulin-like Growth Factor-1 and Insulin-like Growth Factor Binding Proteins from Differentiated Skeletal Muscle Cells. J. Biol. Chem. 1995, 270, 2099-2106. [CrossRef]

49. Ahmad, S.S.; Ahmad, K.; Lee, E.J.; Lee, Y.-H.; Choi, I. Implications of Insulin-Like Growth Factor-1 in Skeletal Muscle and Various Diseases. Cells 2020, 9, 1773. [CrossRef]

50. Sandri, M. Signaling in Muscle Atrophy and Hypertrophy. Physiology 2008, 23, 160-170. [CrossRef]

51. Frost, R.A.; Lang, C.H. Multifaceted Role of Insulin-Like Growth Factors and Mammalian Target of Rapamycin in Skeletal Muscle. Endocrinol. Metab. Clin. North Am. 2012, 41, 297-322. [CrossRef]

52. Coleman, M.E.; DeMayo, F.; Yin, K.C.; Lee, H.M.; Geske, R.; Montgomery, C.; Schwartz, R.J. Myogenic Vector Expression of Insulin-like Growth Factor I Stimulates Muscle Cell Differentiation and Myofiber Hypertrophy in Transgenic Mice. J. Biol. Chem. 1995, 270, 12109-12116. [CrossRef]

53. Gillery, P.; Leperre, A.; Borel, J.-P. Insulin-like growth factor-I (IGF-i) stimulates protein synthesis and collagen gene expression in monolayer and lattice cultures of fibroblasts. J. Cell. Physiol. 1992, 152, 389-396. [CrossRef]

54. Swasdison, S.; Mayne, R. Formation of highly organized skeletal muscle fibers in vitro. Comparison with muscle development in vivo. J. Cell Sci. 1992, 102, 643-652. [CrossRef] [PubMed]

55. VanDenburgh, H.H.; Karlisch, P.; Farr, L. Maintenance of highly contractile tissue-cultured avian skeletal myotubes in collagen gel. Vitr. Cell. Dev. Biol.-Anim. 1988, 24, 166-174. [CrossRef]

56. Zdzieblik, D.; Oesser, S.; Baumstark, M.W.; Gollhofer, A.; König, D. Collagen peptide supplementation in combination with resistance training improves body composition and increases muscle strength in elderly sarcopenic men: A randomised controlled trial. Br. J. Nutr. 2015, 114, 1237-1245. [CrossRef] [PubMed]

57. Lee, K.; Yi, C.-W.; Lee, S. The effects of kinesiology taping therapy on degenerative knee arthritis patients' pain, function, and joint range of motion. J. Phys. Ther. Sci. 2016, 28, 63-66. [CrossRef]

58. Park, J.-S.; Yoon, T.; Lee, S.-H.; Hwang, N.-K.; Lee, J.-H.; Jung, Y.-J.; Lee, G. Immediate effects of kinesiology tape on the pain and gait function in older adults with knee osteoarthritis. Medicine 2019, 98, e17880. [CrossRef] [PubMed]

59. Ostrander, R.V.; Leddon, C.E.; Hackel, J.G.; O'Grady, C.P.; Roth, C.A. Efficacy of Unloader Bracing in Reducing Symptoms of Knee Osteoarthritis. Am. J. Orthop. 2016, 45, 306-311.

60. Thoumie, P.; Marty, M.; Avouac, B.; Pallez, A.; Vaumousse, A.; Pipet, L.P.T.; Monroche, A.; Graveleau, N.; Bonnin, A.; Ben Amor, C.; et al. Effect of unloading brace treatment on pain and function in patients with symptomatic knee osteoarthritis: The ROTOR randomized clinical trial. Sci. Rep. 2018, 8, 10519. [CrossRef]

61. Kuyinu, E.L.; Narayanan, G.; Nair, L.S.; Laurencin, C.T. Animal models of osteoarthritis: Classification, update, and measurement of outcomes. J. Orthop. Surg. Res. 2016, 11, 1-27. [CrossRef] 
62. Franke, T.F.; Hornik, C.P.; Segev, L.; Shostak, G.A.; Sugimoto, C. PI3K/Akt and apoptosis: Size matters. Oncogene 2003, 22, 8983-8998. [CrossRef] [PubMed]

63. Strzalka, W.; Ziemienowicz, A. Proliferating cell nuclear antigen (PCNA): A key factor in DNA replication and cell cycle regulation. Ann. Bot. 2010, 107, 1127-1140. [CrossRef] [PubMed] 